\title{
Ein Jahr Anaphylaxie-Register
}

\author{
Das Allergie-Centrum-Charité in Berlin hat vor einem Jahr ein Melde- \\ system ins Leben gerufen, mit dem möglichst alle anaphylaktischen \\ Reaktionen im deutschsprachigen Raum erfasst werden sollen. Prof. \\ Dr. Margitta Worm, Leiterin des Anaphylaxie-Registers, erläutert die \\ Hintergründe und stellt erste Ergebnisse vor.
}

- Ans Aus welchem Grund haben Sie das Anaphylaxie-Register initiiert? Worm: Wir wollen wissen, wie häufig Anaphylaxien in Deutschland auftreten und wie die Patienten versorgt werden. Diese Informationen helfen, die Versorgung von Betroffenen mittel- bis langfristig zu verbessern.

Welche Informationen werden abge-
fragt? Worm: Unser Ziel ist es, möglichst alle betroffenen Patienten zu erfassen. Dabei erfragen wir die Symptome, die Auslöser, die Kofaktoren, die durchgeführte Diagnostik und Therapie sowie eingeleitete Präventionsmaßnahmen.

\section{Gibt es rechtliche Probleme wegen des - Datenschutzes?}

Worm: Wir haben ein Datenschutz- sowie ein Ethikvotum für die Datenerfassung eingeholt. Die Daten werden pseudonymisiert und können nachträglich von dem behandelnden Zentrum wieder entschlüsselt werden.

Wie wird das Anaphylaxie-Register
- finanziert? Worm: Das Register wird derzeit mit Mitteln aus der Industrie unterstützt. Aktuell fördern uns die Unternehmen Allergopharma, Schwarz Pharma und Bencard. Darüber hinaus sind wir bemüht, die Finanzierung mittelfristig durch Einwerbung öffentlicher Gelder auf eine breitere Basis zu stellen.

$?$ Sie haben im November 2007 die Ein- Jahres-Daten des Anaphylaxie-Registers vorgestellt. Über wie viele Datensätze verfügen Sie bisher und was zeigen diese? Worm: Wir haben bis zum 21. November 462 Reaktionen erfasst, davon 62 bei Kindern und 400 bei Erwachsenen. Zwei Fälle führten zum Tod. Bei Kindern sind die häufigsten Auslöser Lebensmittel, vor allem Erdnüsse und Nüsse, gefolgt von Insektengiften. Bei den Erwachsenen stehen als Auslöser die Insektengifte an erster Stelle. Es folgen Medikamente, wie etwa Schmerzmittel und Antibiotika, sowie Lebensmittel, zum Beispiel Krustentiere und Sojabohnen.

Die Versorgung erfolgte zu fast 50\% durch Notärzte. Aber auch die Patienten selbst sowie die Angehörigen und Hausärzte spielen bei der Notfallversorgung eine Rolle. Die Art der Notfallmedikation war bei knapp 200 Patienten nicht bekannt, beispielsweise weil sie das Präparat nicht kannten. Bei den eingesetzten Medikamenten dominierten Glukokortikoide und Antihistaminika. Adrenalin, das wir bei schweren Anaphylaxien bevorzugen, ist hingegen unterpräsentiert. Hier könnte man künftig durch eine verbesserte Aufklärung die Versorgungssituation verbessern. Darüber hinaus haben wir festgestellt, dass bei etwa einem Drit-

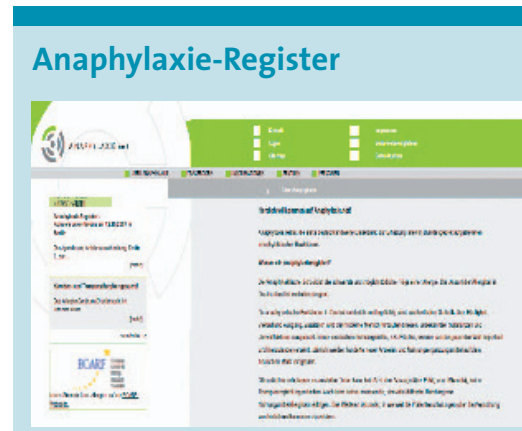

Das Anaphylaxie-Register hat inzwischen zwei Validierungsschritte hinter sich, bei denen der Fragebogen jeweils optimiert wurde. An der Datenerhebung beteiligen sich derzeit 46 allergologische Zentren aus Deutschland, Österreich und der Schweiz. Der Fragebogen ist überwiegend zum Ankreuzen, um das Ausfüllen einfach zu gestalten. Die Eingabe erfolgt über die Website www.anaphylaxie.net in einem durch Passwort geschützten Bereich.

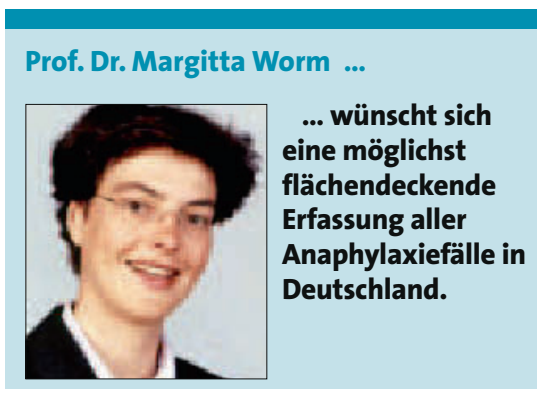

tel der Betroffenen bereits mehrfach Anaphylaxien aufgetreten sind. Für diese Personen sind Prävention und Schulungen sehr wichtig. Schließlich hat sich gezeigt, dass Kofaktoren eine wichtige Rolle spielen und nicht selten mehrere Faktoren zusammenwirken. Zu den wichtigsten gehören Medikamente wie $\beta$-Blocker und Acetylsalicylsäure sowie Herz-Kreislauf-Störungen oder klassische Inhalationsallergien.

Ist es geplant, die Sammlung der Daten - auf andere Kliniken, Notärzte oder niedergelassene Kollegen auszuweiten?

Worm: Da wir bislang nur wenig pädiatrische Zentren haben, bemühen wir uns vor allem, Fälle bei Kindern künftig vollständiger zu erfassen. Außerdem bieten wir die Beteiligung niedergelassener Ärzte zukünftig an. Darüber hinaus führen wir derzeit Befragungen von Notärzten durch. Wir erheben diese Daten jedoch separat, um eine Unterscheidung beispielsweise zwischen allergologischen Zentren und Praxen sowie Hausärzten zu ermöglichen. Interessierte Kollegen schreiben eine E-Mail über die Website des Registers, wir melden uns dann. An die teilnehmenden Ärzte versenden wir regelmäßig Newsletter mit aktuellen Daten des Registers.

3 Wel Welche weiteren Schritte haben Sie für das Anaphylaxie-Register geplant? Worm: Wir wollen das Register in den nächsten Jahren weiter ausbauen. Wir planen Veröffentlichungen und Informationsveranstaltungen. Zudem ist die Vernetzung mit europäischen Zentren vorgesehen, um europaweit Daten erheben zu können.

Vielen Dank für das Gespräch. 